\title{
METHODS AND TOOLS TO ENJOY AND TO STUDY INACCESSIBLE HERITAGE
}

\author{
M. Capone ${ }^{\mathrm{a}}$, M. Campi ${ }^{\mathrm{a}}$ \\ a Diarc, Dept. of Architecture, University of Naples Federico II, 80134 Napoli ITALY- mara.capone@unina.it, \\ campi@unina.it
}

Commission WG V/2

KEY WORDS: $360^{\circ}$ Panoramas, 3D Modeling, 3D PDF, Cultural Heritage, 3D Database, Laser scanning.

\begin{abstract}
:
Our research on a multi-purpose survey of cultural Heritage located in UNESCO Historical Centre of Naples has the following goals: to test some innovative strategies to improve public enjoyment for inaccessible sites; to explore the use of some interactive systems to study heritage in remote; to explore how to access the information system through AR applications. In this paper we are going to focus on comparison between interactive system to access 3D data and photogrammetric processing of panoramic images. We investigated on: a. the use of $360^{\circ}$ panorama for $3 \mathrm{D}$ restitutions; b. the use of $360^{\circ}$ panorama as an interface to $3 \mathrm{D}$ data to extract real 3D coordinates and accurately measure distances; c. the use of 3D PDF to access a 3D database.
\end{abstract}

\section{INTRODUCTION}

The development of ICT is rapidly changing the communication system making it necessary testing Digital Media to find new ways of enjoying, valorisation and managing of Historical Sites, especially when they are mostly inaccessible to public.

Thanks to an agreement between Superintendence for Cultural Heritage, DiArc, Department of Architecture, University of Naples Federico II, and the Interdepartmental Centre Urbaneco, the research team has started a multi-purpose survey of many Churches located in UNESCO Historical Centre of Naples. The G-BEY (Gap BEYond) is a research about an integrated platform for knowledge, valorisation and fruition of the denied artistic and architectural heritage of the city of Naples. In this project, began March 1, 2014 and that will end on 31 December 2015, we are developing a database that will be a research tool for students and scholars and some other applications that will able the virtual tour of Heritage that otherwise could be denied. Generally, we can use some different survey techniques to have 3D data and we can use some different methods to serve them to users but the choice depends on the aim.

We can choose obtaining 3D data from laser scanner or using Image-based modelling techniques, to process point cloud to have a 3D model or to use a 3D model from images to have 3D data. We don't think one method is better than the other but we have to decide what is the best for our needs.

The research has the following goals: to test some innovative strategies to improve public enjoyment for inaccessible sites; to explore the use of some interactive systems to study cultural heritage in remote; to explore how to access the information system through AR applications. So our first problem to solve is to look for an easy way to manage 3D data in a database but we are also looking for an easy way to obtain 3D data. We are exploring the possibility of using $360^{\circ}$ panoramas to obtain $3 \mathrm{D}$ data because within cultural heritage applications is increasingly being applied.

In this paper we are going to focus on comparison between interactive system to access $3 \mathrm{D}$ data and photogrammetric processing of panoramic images.

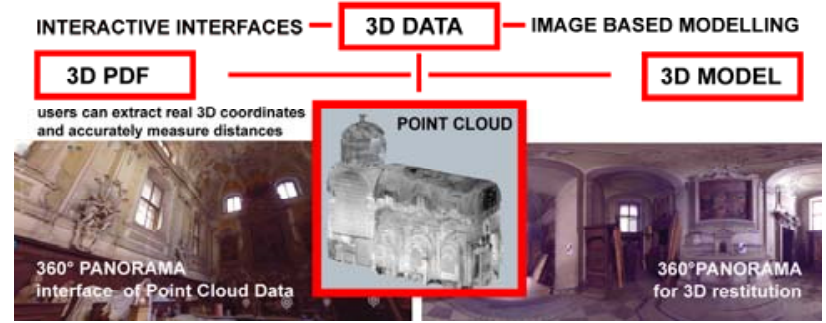

Figure 1. Relationships between 3D data and 3D model: from a point cloud you can have a $3 \mathrm{D}$ model or a $360^{\circ}$ panorama that could be used as interactive interface to access 3D data. From $360^{\circ}$ we can have $3 \mathrm{D}$ data and $3 \mathrm{D}$ model to interact with them.

We are investigating on:

a. the use of $360^{\circ}$ panorama for $3 \mathrm{D}$ restitutions;

b. the use of $360^{\circ}$ panorama as an interface to $3 D$ data to extract real 3D coordinates and accurately measure distances;

c. the use of 3D PDF to access a 3D database.

The main target is to explore the power of multiple interactive representation methods to enjoy and to study CULTURAL Heritage in remote, to offer to researchers (historians, administrative technicians, etc.) even without any particular competence in using technical interfaces, such as the ones for using data performed by scanner lasers sensors or image based modelling techniques. In particular, the systems based on using spherical images can be adopted to share easily 3D data on simple browsers platform. The aim expressed in the paper proposed is to generate an interactive database, which offer information on different layers, each for any different kind of potential user. The goal is not only to offer a visual experience of the heritage analyzed, but to give the possibility to take directly other information as, for example, to measure parts and elements of the architecture.

This case happens very often especially when different kind of researchers have to approach to the same historical heritage for several study aims. The need to have particular information to 


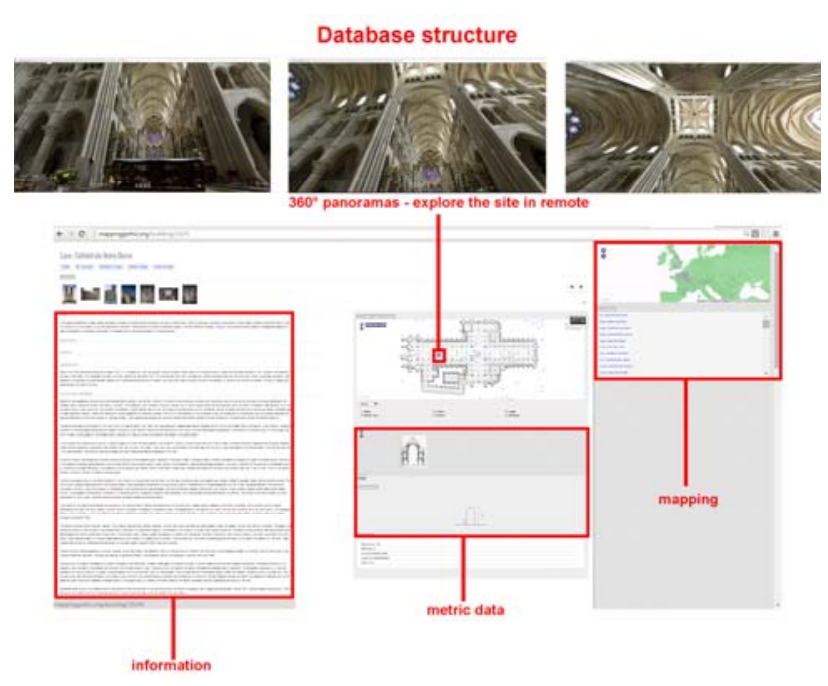

Figure 2. Mapping Gothic France is an interesting cultural Heritage application, it's a database based on the systematic use of $360^{\circ}$ panoramic imagery.

endorse some hypothesis could oblige to catch some dimensional measures that could need difficult surveys.

The possibility to make this operations in remote mode could give an interesting spectrum of possibilities of investigation.

In order to give as much information as possible to users, the database has to integrate different systems of data, obtained by different methodologies, to join a wider range of cultural elements: the systems based also on the interactive use of $3 \mathrm{D}$ models easily navigable, such as 3D PDF file, could offer a solution to the problem. In this way, it is possible to have multiple acquisitions that can be compared to a double goal:

a. to take the best type of information to match with the capability and skill of the final user, users can directly extract 3D real coordinates and accurately measure distances;

b. to make a comparison among different methodologies and techniques, so to define a system of data that takes advantage from all of them and gives a result with the most high degree of accuracy, to create a system more useful and more adequate for maintenance, restoration or analyzing Heritage.

\section{APPLICATIONS $360^{\circ}$ PANORAMIC IMAGES FOR CULTURAL HERITAGE}

Within cultural heritage applications $360^{\circ}$ panoramic imagery is increasingly being applied. Immersive imaging techniques can be used to provide virtual tours.

Taking a series of photos in sequence, so ideally cover the surface of a sphere circumscribed to the pinch point, and using one of several available stitching software, you get a single panorama, $360^{\circ} \times 180^{\circ}$, which represents the whole visible sphere (Szeliski 1997).

This panoramic image can be explored by PC, smartphones and tablets using dedicated software, you can produce interactive videos that can be linked to maps, hotspots, and dynamic text.

The systematic use of panoramic images within a database allows you to gain a thorough understanding especially linked to the perceptual aspects. But if you want to go beyond the look because you want to study and not only to look, you have to be able to measure the object.

We have two different solutions to this problem: we can use $360^{\circ}$ panoramas as a user interface to access $3 \mathrm{D}$ data or we can use them for 3D restitution, the first solution is suitable for all users, while the second is just for experts.

\subsection{The use of $360^{\circ}$ panoramic images for $3 D$ restitutions}

The use of spherical photogrammetry to produce 3D models is a method that could be used to study heritage in remote or when it is destroyed and you have only $360^{\circ}$ panoramic images to reconstruct the situation before. When we have more than one panoramic image it is possible to obtain 3D data: using some of the new experimental systems based on the combined use of the interactive Image-based modeling and the spherical photogrammetry we can produce a 3D restitution (Fangi, Wahbeh, 2013).

With this method you can produce a high resolution textured 3D model using the so called Interactive Panoramic ImageBased Modeling based on the use of 3D modeler as survey instrument. (Fangi, Wahbe, 2013).

Actually, we are exploring the potentiality of this method in two researches, in G-BEY and in another research about the geometry of Gothic Vaults.

Studying the gothic French cathedrals we have found a very interesting database, Mapping Gothic France, developed by the Media Center for Art History in the Department of Art History and Archaeology at Columbia University, the Visual Resources Library at Vassar College, and the Columbia University Libraries, where the cathedrals are systematically documented using $360^{\circ}$ panoramic images (Figure 2).

Two different needs lead us to look for the best technical solution to use the $360^{\circ}$ panoramic images to serve or to have metric information. In the first case, in G-BAY research, we are the designers of database on the contrary we are the users of Mapping Gothic France.

First of all we have done a state-of-the-art of a so called panoramic Spherical Photogrammetry (PSP) (Fangi, 2007), our aim is to explore the potential of this imaged-based method of restitution to study gothic French cathedrals from $360^{\circ}$ panoramic images available on the website or to use them as an economic survey method, in place of laser scanner, to produce 3D model for G-BAY database.

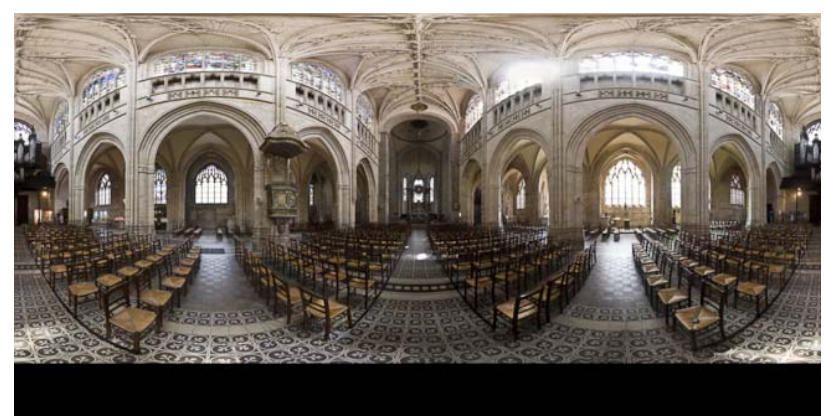

Figure 3. Mapping Gothic France. High resolution equirectangular projection from database.

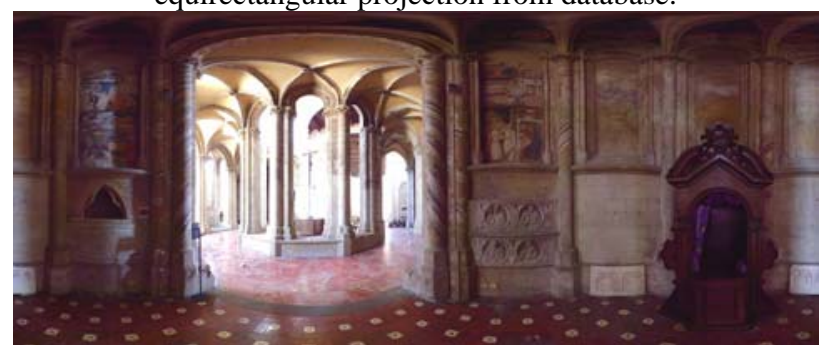

Figure 4 . Naples S. Lorenzo Maggiore. Equirectangular projections from laser scanner. 
In our research, that will end in December 2015, we are going to explore the potential of this system.

From the theoretical point of view the geometry of the epipolar lines in the whole image of the spherical panorama has been just analysed and the correctness of the algorithm has been assessed (Fangi, Nardinocchi, 2013.) for this reason now you have to work on procedure to make automatic the restitution process. Spherical Photogrammetry, PSP, pipeline is based on recent advances in automatic restitution processes. PSP could be a very good system for cultural heritage metric documentation especially when we have to document many sites, it is the case of G-Bay, a database in which we have to put information on more than 800 churches of Naples. The main advantages of this method are the speed of the survey, the low cost of the equipment and the completeness of the acquired information, but the orientation and restitution procedures are still fully manual (Fangi, Nardinocchi, 2013).

We can generate panoramic images in different way but we can use these images for measurement only when they are made by using a tripod and a spherical head expressly designed for panoramic imaging, allowing the coincidence of the centre of views of the images. Using some of the most popular stitching software we can compensate both for radial distortion and for residual horizontal and vertical shift offsets and produce a panoramic image that can be used for a 3D restitution.

Actually the method is based on classical photogrammetry principles: orientation of multiple panoramas and manual 3D object reconstruction.

Until automated PSP processing becomes available recent experimentation in the manual restitution are generally based on the use of Image Based Modeler software, such us Autodesk Image Modeler, that allow directly produce 3D model from oriented photos and panoramas. Calibration, modeling, and texturing are the three-steps of this system but there are some problems in modeling tools, thus sometimes we need to use other 3D model software (Cannella, 2013). To calibrate the equirectangular panoramas you need 6 points well recognized in the images, it's better more than 6 , and to get more accurate 3D model you should also use some known geometrical features or, if it's possible, the coordinates of some points. The expertise of the operator is very important to produce an accurate 3D model based on the homologous points search, as a matter of fact that image matching may generate a very great errors as well as an incorrect reading of the geometries.

In our researches we are going to produce 3D model of San Lorenzo Maggiore in Naples from $360^{\circ}$ panoramas using Image Modeler. The comparison between 3D data from laser scanner survey will allow us to assess different geometric accuracies coming from different sources of information, also in relation to processing time to produce 3D model.

We are also testing this method in the research about Gothic French cathedrals. In this case we are interesting in the study of geometric configuration of the vaults and we think that the accuracies of the 3D model produced it could be good for our aims and errors in measurement are within acceptable tolerances required by research in progress.

\subsection{The use of $360^{\circ}$ panoramic image as interface to $3 D$ data}

In recent years the advances in laser scanner technologies has allowed fusion of digital panoramic camera data with laser scanner data, this has strongly contributed to widespread use of $360^{\circ}$ panoramic images. We have used a FARO Focus 3d Laser Scanner for our survey, that allows to export each individual scan as a panoramic image.
The spherical images from laser scanner could be also adopted to share easily 3D data on simple browsers platform. SCENE WebShare Cloud, FARO, offers a comprehensive service package to provide users with simple access to 3D documentation. The structure for WebShare is produced through automated software and can be readily made available on an intranet or internet via Webshare Server (which is free). This enables a publishing project and multi-user browser using panoramic images (one for each scan) with simultaneous display of position and orientation in plan.

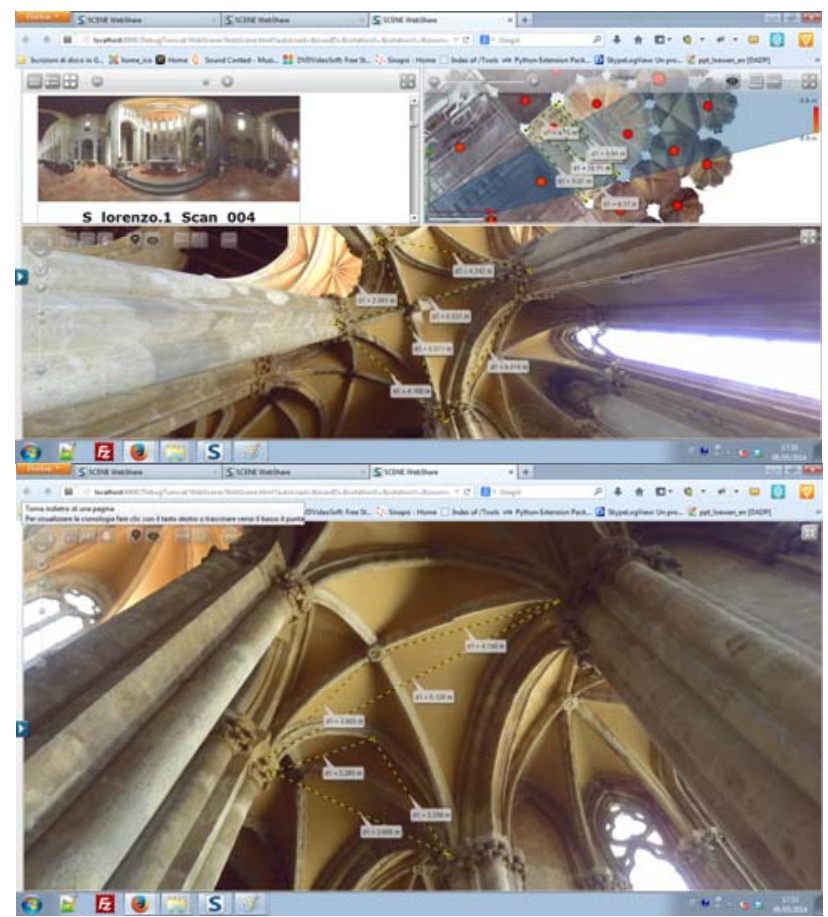

Figure 5 . Naples S. Lorenzo Maggiore. SCENE WebShare: using this system $360^{\circ}$ immersive images could be considered a user interface to access 3D data.

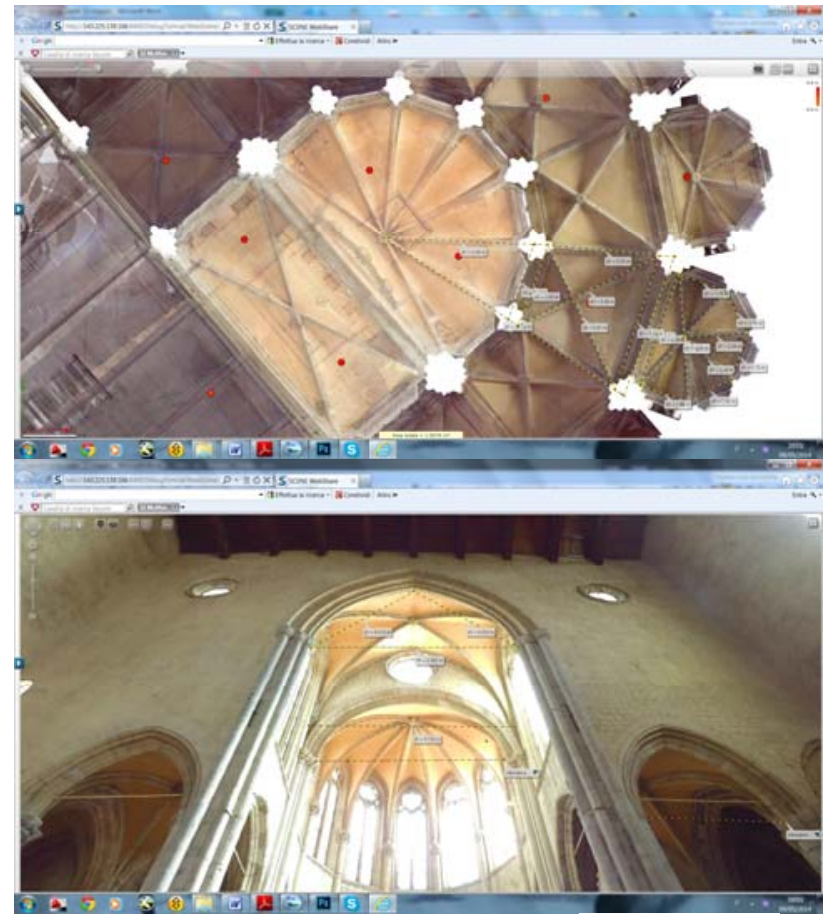

Figure 6 . Naples S. Lorenzo Maggiore. Survey data from SCENE WebShare Cloud. 


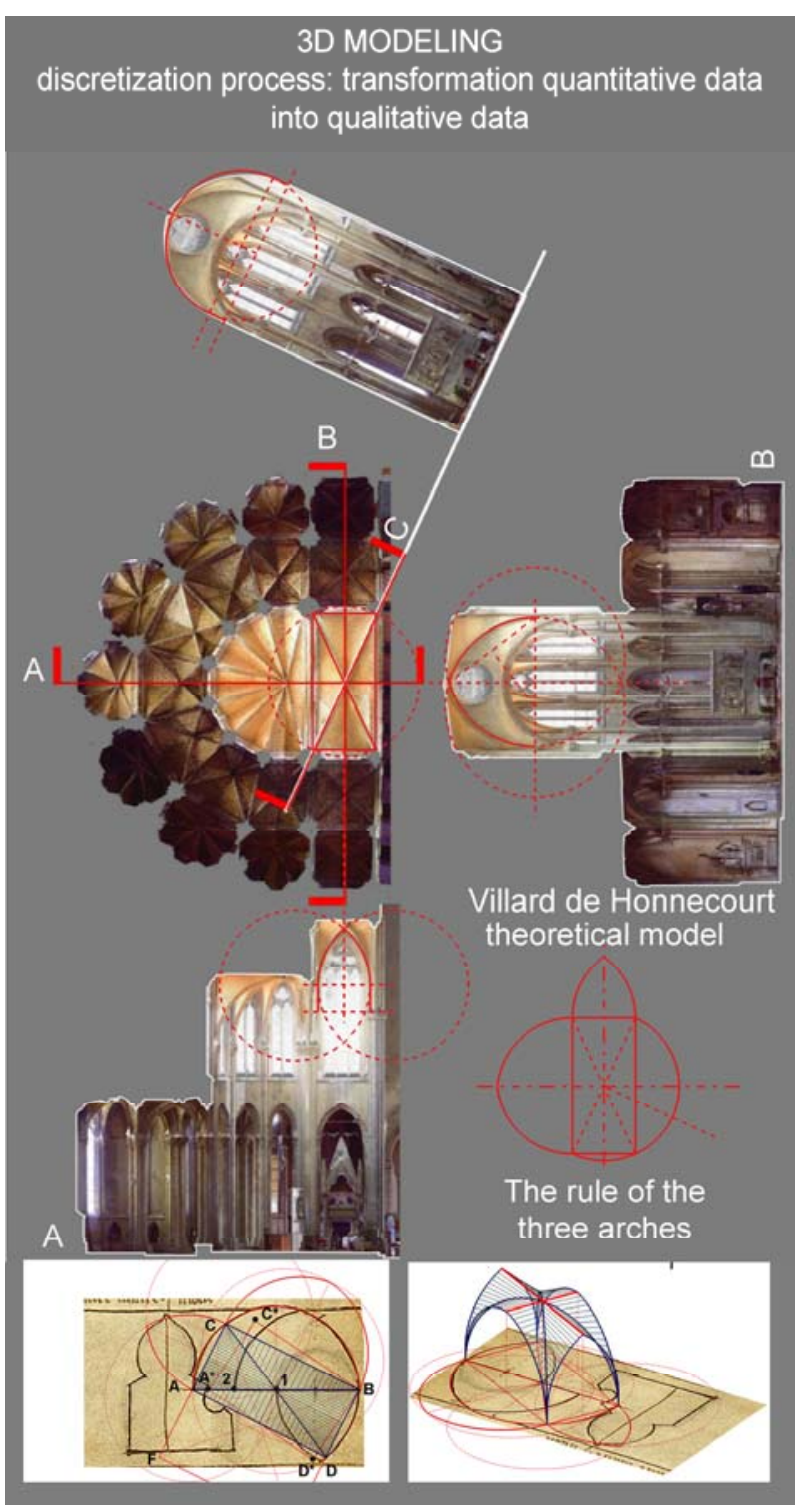

Figure 7. S. Lorenzo. Comparison betweens model from 3D laser scanner point cloud and theoretical model.

These aren't simple panoramas because they are produced by 3D dimensional scan, for this reason, using this system, it's possible conducting measurements directly on the panoramic images (Figures 5, 6).

We are going to use the WebShare structure in our database, in this way, $360^{\circ}$ panoramas produced from laser scanner could be considered a user friendly interfaces to interact with 3D data. In some cases this system could be very useful for scholars, that need to measure in order to verify their hypotheses.

In our research about Gothic cathedrals we have used this method to study the geometric configuration of Church of San Lorenzo Maggiore in Naples.

Our target is to understand the transformation of gothic features from French to Naples. To study the geometric vaults system, we have produced three models: one theoretical model based on the study of treatises, one surveying data from SCENE WebShare Cloud and the last one using data from laser scanner survey (Figures 7, 8). In this way we are able to test the different geometric accuracies coming from different sources of information and to verify our critical hypotheses. In fact, from the study of Viollet-le-Duc and Villard de Honnecourt

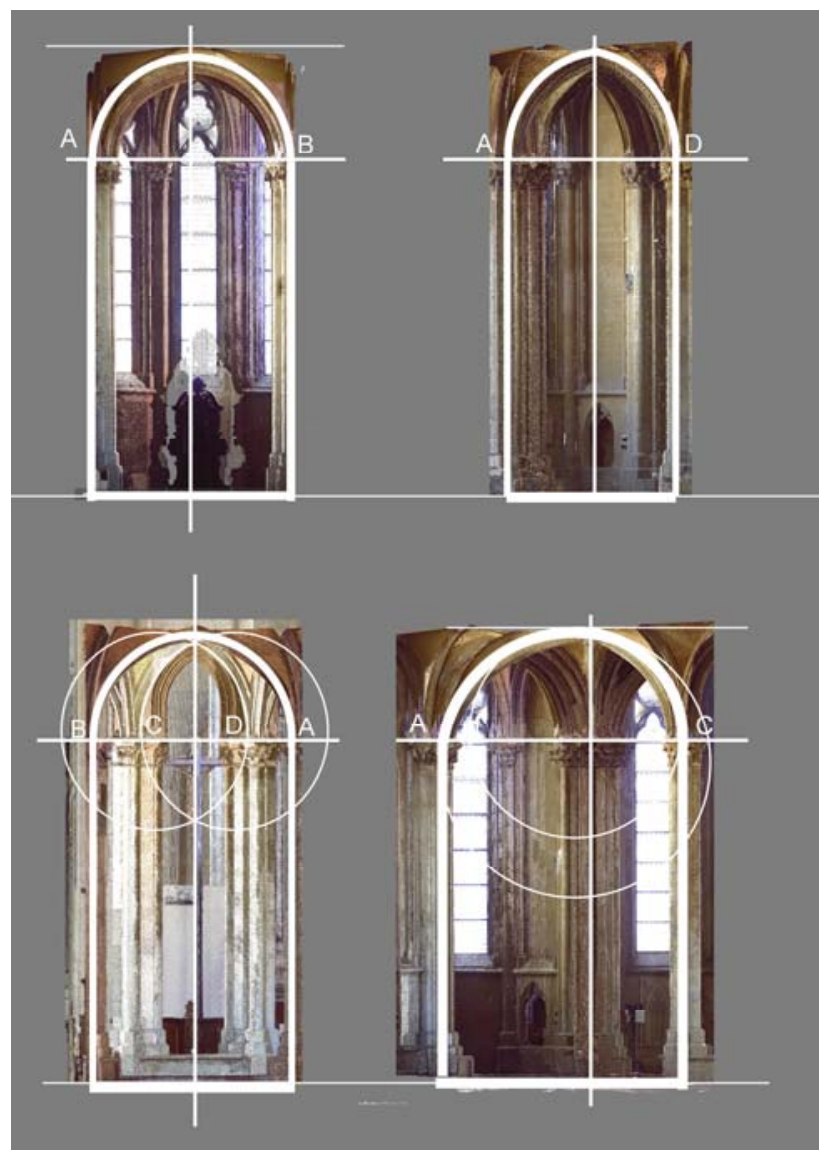

Figure 8 . Naples S. Lorenzo Maggiore. Gothic vaults geometry from laser scanner data.

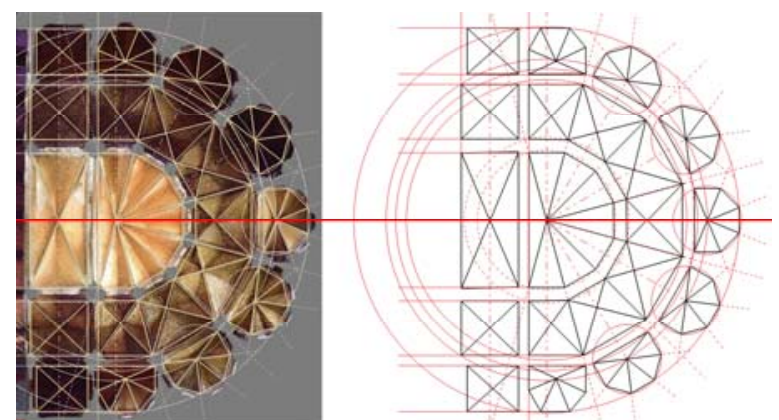

Figure 9. S. Lorenzo, Geometry from laser scanner point cloud.

we can produce a 3D model based on the application of some rules, for example the three arcs rule of Villard, and then we can check if these rules have been followed or not (Figure 7).

If you consider the gothic trapezoidal vaults in S. Lorenzo ambulatory, you can built a model according to treatises. You can produce some different models, for example you can assume that diagonal ribs $\mathrm{AC}$ and $\mathrm{BD}$ are semicircular arches and the $\mathrm{AD}, \mathrm{BC}$ and $\mathrm{DB}$ are pointed arches with the same radius of curvature of diagonal arches. In this way we can verify producing $3 \mathrm{D}$ model based on these hypotheses that the keystones of the arches are at different heights (Figure 10).

To understand what kind of rules has been used in S. Lorenzo, we have crossed the point cloud to verify the arches nature and we have surveyed that the diagonal arches AC and BD aren't semicircular arches but they are two centers arches, while $\mathrm{AB}$ is a semicircular arches and $\mathrm{AD}, \mathrm{BC}$ and $\mathrm{DC}$ are pointed arches with same radius of $\mathrm{AB}$ (Figure 11). 

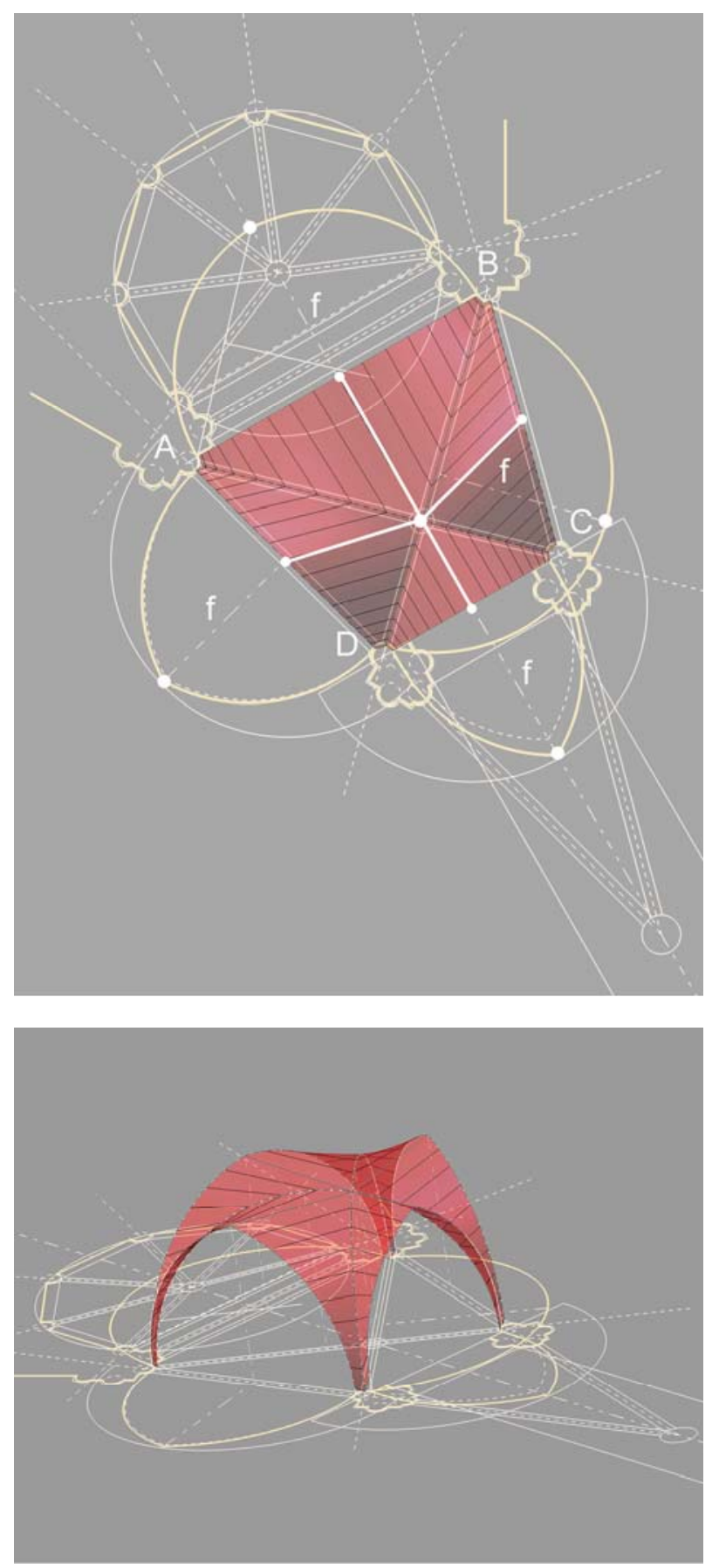

Figure 10. S. Lorenzo, 3D Geometry model from theoretical hypotheses

The comparison between 3D model from laser scanner and 3D model from WebShare allows us to value two different manual methods 3D model production, while the comparison with the theoretical model allows us to deepen knowledge of the cultural heritage. As a matter of fact that, survey isn't only a metric documentation method but it is a critical analysis tool.

\section{3D PDF: AN INTERFACE TO ACCESS 3D DATA}

In our researches we are going to use 3D model to produce an interface to access 3D data. Interfaces are generally all physical or virtual "devices" that allow exchange information between two or more different systems.

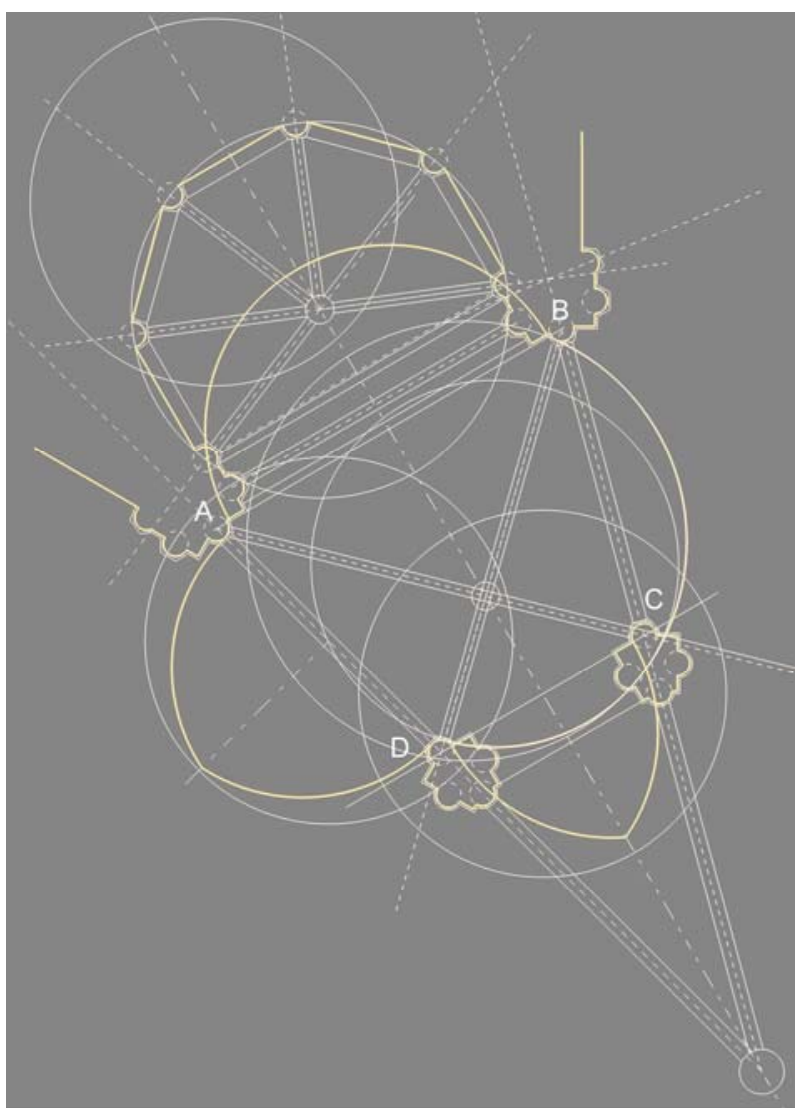

Figure 11. S. Lorenzo, Geometry model from laser scanner survey

We can define "user interface", everything allows humans to interact with the machine. So the concept of interface is tightly connected to interactivity.

We can access to information systems using graphical interfaces, a set of "objects" graphics that allow to interact with a database that has specific contents and aims.

The conventional interfaces, based on the use of interactive graphics essentially made by keys, are increasingly being replaced by iconic systems develop around the principle of WYSIWYG (What You See Is What You Get), interfaces that represent objects in their real configuration and not using an abstract code.

The choice of the interface depends on the structure and the goals of the database, for this reason it can be designed in different ways.

Research is increasingly oriented towards identifying structures that stimulate the cognitive mechanisms to facilitate the transformation of information into knowledge. In these play a key role designing interfaces that allow the user to access information in a natural and instinctive way.

Usability, Comprehensibility, Originality and Agreeableness are the basic requirements in the design of an interface that is able to reflect the aim of the information system which it refers to, trying not to reduce the interaction to a simple preestablished path.

Interactivity is the difference between analogical and digital media, and the interaction between man and computer system evolves from multimedia to multisensory, developing interfaces that let us interactively explore virtual environments and interact with 3D objects. 

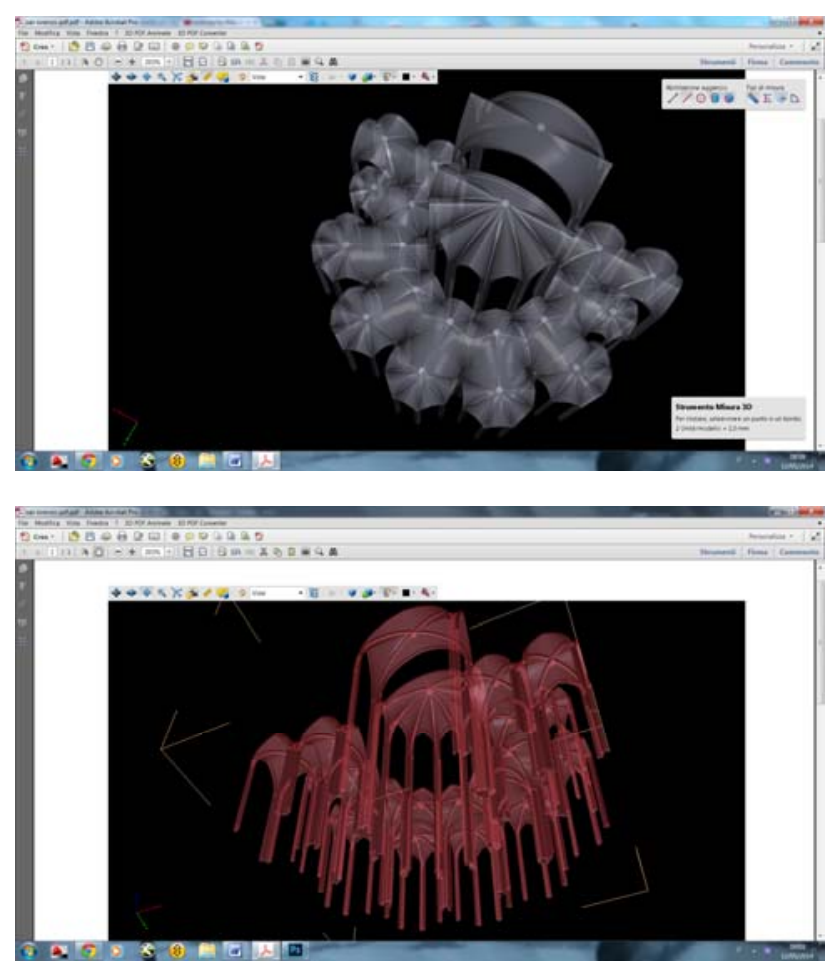

Figure 12. Naples S. Lorenzo Maggiore. 3D PDF: a tool to spread knowledge.

For this reason to produce this kind of interface you have to produce a 3D model and you can do it in several ways. We are going to explore these ways to understand the differences about the accuracy and the visualization modes, our aims is to be able to choose the best method in relation to the objectives.

\subsection{D model for serving data to users}

Recent developments in ICT, 3D technologies and measurement instrumentation open new perspectives for Cultural Heritage documentation.

The great advances in $3 \mathrm{D}$ modeling allow us to design a multimedia database not only for wide public but mainly for scientific research proposes.

3D model construction is the first sep to produce a user friendly interface for serving data, thus the representation code should be chosen in relation to the database goals. When we design a 3D interface we have to decide if it's better to use a realistic representation or an iconic one, if we want to show the look of the object or his hidden structure to spread its cultural meaning. Generally, the aim of 3D modelers is to produce a photorealistic $3 \mathrm{D}$ model that sometimes is a sort of a clone of the real object because they are often more interested in the technological aspects than to the cultural ones.

A multimedia database with a realistic 3D model isn't so interesting for our case studies because we can see the Churches using the $360^{\circ}$ panoramic images better then a virtual simulation of them.

You can produce 3D model in some different ways, it is possible to use automatic processing techniques, such as automated 3D reconstruction from terrestrial images, or you can produce a 3D model using manual techniques and if you need $3 \mathrm{D}$ realistic model it's better to use imaged-based techniques then range-based techniques.

3D Laser Scanner actually creates high resolution point clouds in only a few minutes but data processing for modeling demands a lot of time and work.

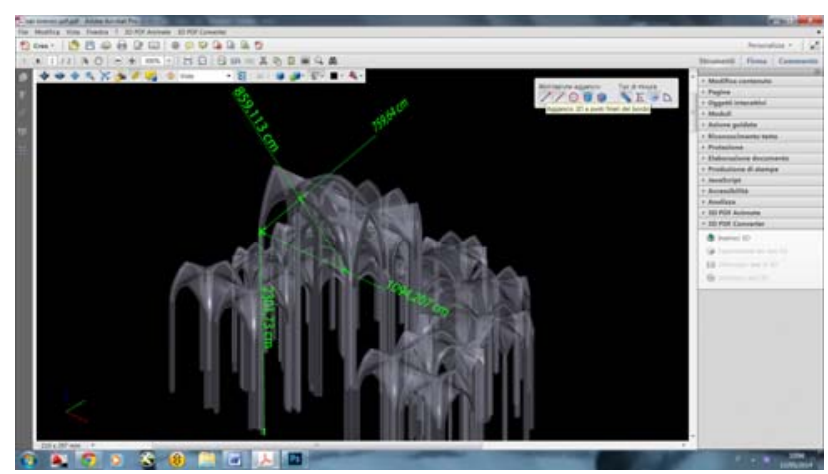

Figure 13 . Naples S. Lorenzo Maggiore. 3D PDF: testing 3D Measurement Tools.

The research trend is to find systems for 3D automated reconstruction from laser scanner data, based on intelligent algorithms (El Meouche, 2013) but these systems are still in the experimental stage.

In our research we are going to use $3 \mathrm{D}$ data to generate a $3 \mathrm{D}$ geometric model based on discretization process in which we have to transform quantitative data into qualitative data, and thus we have to decide how much or how little detail to extract from scans in relation to the research aims.

In our case studies we don't need to use 3D automated reconstruction from laser scanner data because the survey is a knowledge tool and 3D model is generated to produce an interface for serving data to users. In this case, the expertise of the operator is very important to produce an accurate 3D model based on understanding of geometry and the study of the Treaties.

We are going to use this 3D model to generate a 3D PDF and to explore the possibility to use it like a user friendly interface both from generic users and from scholars to acquire information about metric data but not only.

\subsection{The use of 3D PDF to access a 3D database}

One of the main innovations offered by digital technology is the possibility to interact with 3D models independently from software used to produce them.

It is possible to generate a 3D PDF from a 3D modelling program and interact with it using Adobe Acrobat Reader, put it in a presentation in Power Point or in a PDF document (Pletinckx 2011). The aim is to test the communicative potential arising from the possibility to interactively explore 3D models. Interactivity gives the user a level of knowledge certainly different from videos or images that show the object. 3D models can become a user friendly interface to access other information related to it, through the three dimensional display system it is possible to visualize different views of the model, you can link them to text or some others information, such as images, photos, videos and so on.

It is possible to generate a 3D PDF establishing different types of visualizations (opaque, transparent, wireframe ...) that can be easily viewed using Acrobat Reader.

Generally, 3D PDF isn't a photorealistic model but it allows the users to acquire a greater awareness through the interaction with the 3D model.

The use of 3D PDF let us to link to 3D model some others data by layering, named views, action and so on.

The experimentation in this field is to produce models using different forms of visualizations for different communication aims and to develop a measurement tool based on the use of 3D PDF files and Acrobat X Pro. 


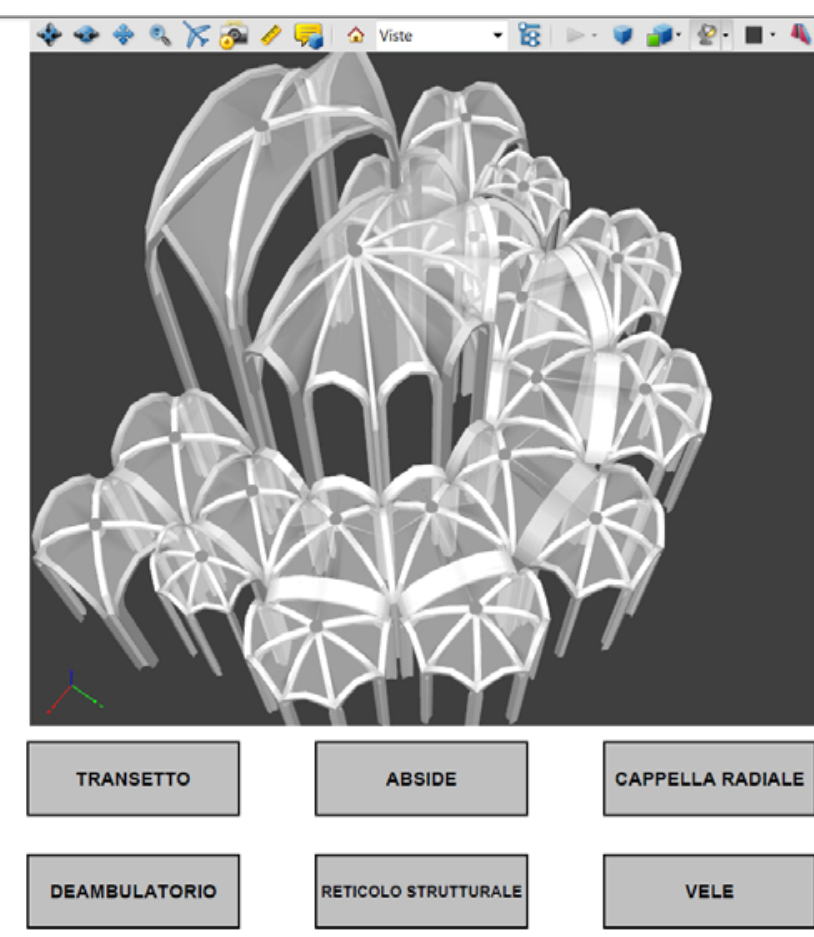

Figure 14 . Naples S. Lorenzo Maggiore. 3D PDF: testing layering tools

It is possible to use the 3D Measurement Tool to measure 3D models. You can create measurements between combinations of points or edges of the 3D model (Figure 13).

The 3D Measurement Tool supports four types of measurements: perpendicular distance between two straight edges, linear distance between two points, the radius of circular edges, and the angle between two edges (or three points). You can associate 3D measurements with specific views. If the default view is active when a measurement is added, a new measurement view is created.

You can also display comments while taking measurements. These comments (also called measurement markups) are preserved after the document is closed.

We are also testing different methods for texturing 3D object to define a visualization code and to choose the best way to spread cultural content for a wide audience and we are testing layering options to link information to 3D model in a 3D PDF.

We are going to use 3D PDF options to explain the building method comparing with the theoretical model based on study of treatises.

In our PDF document, we are able to guide the user linking to a text, some particular views to visualize the object in a certain way to underline some features of it. We created special named view to improve the public enjoyment of 3D model by interactivity and knowledge of the gothic geometry rules. We have decided to show the model not only in a render mode, where the hidden lines are invisible, but we have chosen to visualize the model showing the hidden structure.

We carefully have planed the named views that we considered more useful for visualization and interaction.

There are a lots of benefits to use actions, first of all we can update and expand the database linked to 3D model in an easy way and we improve the access to 3D exploration of the model also for no expert people. One of the most important target of our research is to test a new strategy to improve public enjoyment using 3D PDF files. Another problem to solve is to simplify the 3D model to create 3D PDF file.
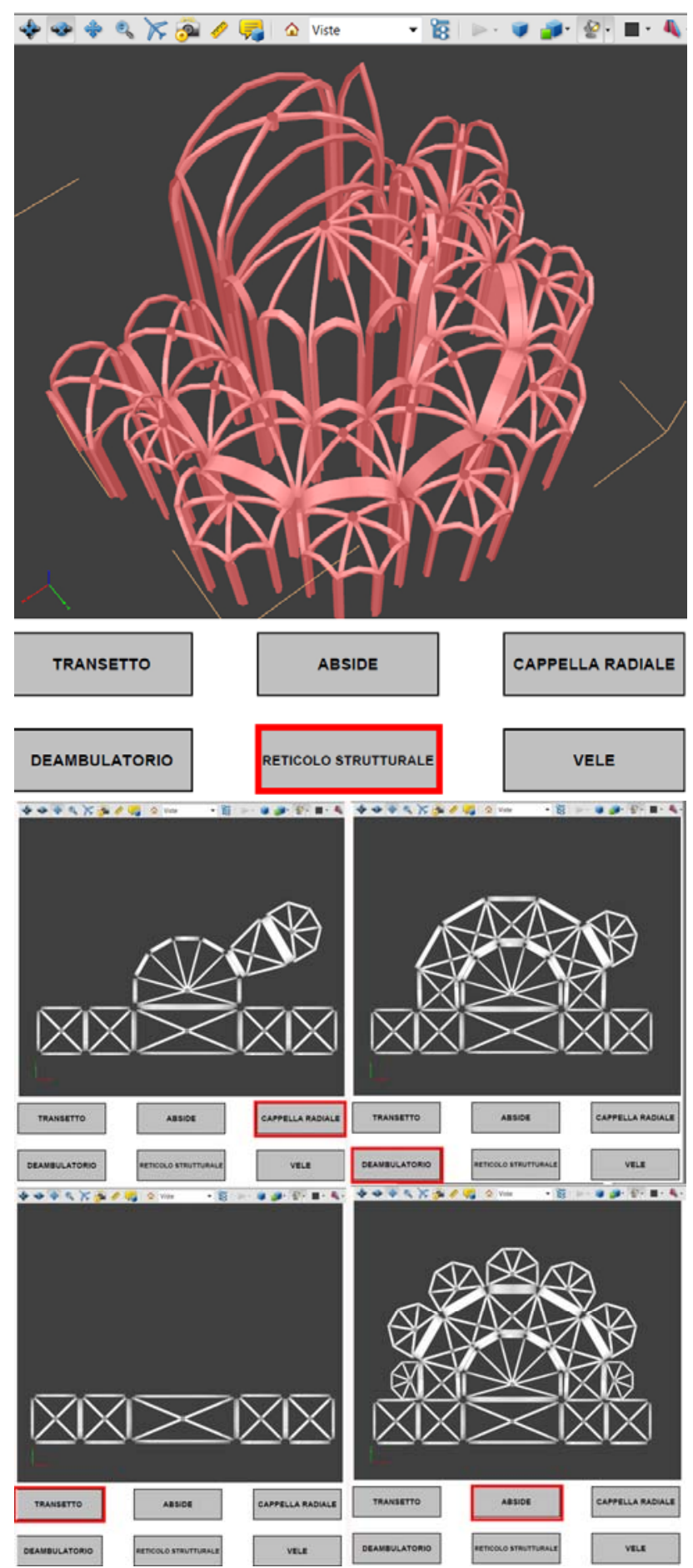

Figure 15 . Naples S. Lorenzo Maggiore. 3D PDF: testing 3D LayeringTools.

We have to reduce the complexity of the 3D model choosing details, texture in an adequate way. The target is to have a 3D model file not too large without losing its communication characteristic.

\section{CONCLUSIONS}

To explore, to know ant to study Cultural Heritage in remote you can use some different systems based on $360^{\circ}$ panoramic images or interactive exploration of $3 \mathrm{D}$ models. Panoramic images are increasingly used for cultural heritage 
documentation, this technique are fast and economic and in recent years it allows to produce high accuracy 3D models.

The main advantage of image based techniques for scholars is the possibility to process these images in any time and to use them according to goals of their own research. For this reasons the $360^{\circ}$ panoramic images are the best way to document architectonical Heritage both for scholars and for common users, they allow to explore places also when they are closed to wide public or when they are simply far. There are several advantages to use panoramic images to measurement but the orientation and restitution methods are sill manual, for this reason they can be used only by experts. On the contrary, the use of $360^{\circ}$ panoramas from laser scanner is a very good system for all kind of users but the 3D measurement tools are not very accuracy, for this reason we think that the system can be used when the target is to verify some hypotheses and you don't need very accuracy measures. Very interesting could be the possibility to download points cloud from that scholars could study heritage in remote.

Interaction with 3D model is a very new way to serve 3D data. To use this method we have to do a 3D model, thus we have to choose the survey technique and the way to produce 3D model, this choice depends on the aims of research, for example if the main goal is to study the heritage you don't need 3D model automated reconstruction from 3D data and you will prefer the manual systems because measuring you will be able to verify your hypotheses or formulate new ones.

Our research is a work in progress, we are especially working on use of $360^{\circ}$ panoramas to survey architecture in remote and to test 3D PDF use as measurement tool, the main goal is to find the best way to serve 3D data both for wide public and scholars.

\section{REFERENCES}

Aguilera D.G., Lahoz J. G. 2006. Laser Scanner or Image-based modeling? A comparative through of San Nicolas Church. In: International Archives of the Photogrammetry, Remote Sensing and Spatial Information Sciences. Dresden, Germany Volume XXXVI - Part 5.

Murray S., Tallon A. 2011. Mapping Gothic France. Media Center for Art History, Department of Art History and Archaeology at Columbia University, the Visual Resources Library at Vassar College, Columbia University Libraries. http://mappinggothic.org

Barazzetti L., Fangi G., Remondino F., Scaioni M. 2010. Automation in multi-image spherical photogrammetry for 3D architectural reconstructions. Proc. of 11th Int. Symposium on Virtual Reality, Archaeology and Cultural Heritage (VAST 2010), 21-24 September, Paris, France, pp. 75-81

Cannella M. 2013. Ratings on the use of the spherical photogrammetry for the 3Dmodels construction: the Oratory of St. Catherine's Parish Church in Zejtun (Malta). In: Disegnare con. Vol. 6, n. 12 Disegnare con la fotografia digitale, a cura di Pablo Rodríguez-Navarro.

Cardaci A., Versaci A. 2013. Image-based 3D modeling vs laser scanning for the analysis of medieval architecture: the case of St. Croce in Bergam. In: International Archives of the Photogrammetry, Remote Sensing and Spatial Information Sciences, Volume XL-5/W2, 2013XXIV International CIPA Symposium, Strasbourg, France.
El Meouche R., Rezoug M., Hijazi Ihab. Dieter M. 2013. Automatic reconstruction of 3D buildings models from terrestrial laser scanner data. In: International Archives of the Photogrammetry, Remote Sensing and Spatial Information Sciences, Cape Town, South Africa. Volume II-4/W1.

Fangi G., Nardinocchi C. 2013. Photogrammetric processing of spherical Panoramas. In: The Photogrammetric Record 28(143), The Remote Sensing and Photogrammetry Society and John Wiley \& Sons Ltd pp. 293-311.

Fangi G., Wahbeh W. 2013. The destroyed minaret of Umayyad Mosque of Aleppo, the survey of the original state. European Scientific Journal. SPECIAL edition vol.4.

Fangi, G., 2007. The multi-image spherical panoramas as a tool for architectural survey. In: International Archives of Photogrammetry, Remote Sensing and Spatial Information Sciences, Athens, Greece - Volume XXXVI-36(5/C53): pp. 311-316.

Jia Li, Yehua Sheng, Ping Duan, Siyang Zhang, Haiyang Lv 2014. Constructing 3D Model Based on Panoramic Images. In: Advances in Computer Science and its Applications. Lecture Notes in: Electrical Engineering, Volume 279, pp 1061-1065.

Pletinckx D. 2011. Europeana and 3D. In: International Archives of the Photogrammetry, Remote Sensing and Spatial Information Sciences. Trento, Italia. Volume XXXVIII-5/W16.

Szeliski, R. and Shum, H., 1997. Creating full view panoramic image mosaics and environment maps. Proceedings of SIGGRAPH. pp. 251-258.

\section{ACKNOWLEDGEMENTS}

The authors are thankful to Raffaele Catuogno and Angela Buonafiglio for the laser scanner survey ant to Lucia Acanfora for the work carried out on the modeling about the S. Lorenzo Church. 